PO-0184 SEASONALITY OF ACUTE BRONCHIOLITIS IN HOSPITALIZATED INFANTS OVER A 4-YEAR PERIOD

${ }^{1} J C$ Flores-Gonzalez, ${ }^{1}$ P Comino-Vazquez, ${ }^{1} M A$ Matamala-Morillo, 'RM Garcia-Ortega, ${ }^{1} \mathrm{~L}$ Garcia-Garcia, ${ }^{1} \mathrm{FJ}$ Dávila-Corrales, ${ }^{1} \mathrm{JJ}$ Perez-Guerrero, ${ }^{1} \mathrm{P}$ Rodriguez-Campoy, 'B Serrano-Moyano, 'E Palma-Zambrana, ${ }^{2}$ AM Lechuga-Sancho. 'Pediatrics Department, Hospital Universitario Puerta Del Mar, Cádiz, Spain; ${ }^{2}$ Cadiz University, Hospital Universitario Puerta Del Mar, Cádiz, Spain

\subsection{6/archdischild-2014-307384.845}

Background and aims Bronchiolitis is the most common lower respiratory infection in infants and a leading cause of hospitalizations in this age group. To describe the rhythm and seasonality of the yearly epidemics of bronchiolitis in hospitalised children.

Methods Epidemiological analysis of patients who were admitted to our hospital with bronchiolitis, over an 4-year period, from 2008 to 2012. The epidemic onset and conclusion was established according to the first and last cases per months, respectively.

Results Data was collected from 389 patients, mean age: 59 days (5-739), 59\% male. The mean length of stay was 5,9 days $(2-71)$. There was no risk factor in $87 \%$ (10\% premature and $1,5 \%$ heart disease). The clinical score at hospitalisation was mild (4\%), moderate (43\%) and severe (2,8\%). The RSV was positive $67 \%$. Epidemics begin in October (50\%) and November (25\%); the highest peak was observed in January, February and December and its conclusion varies between March and July. There were no cases in August and September.

Conclusion Bronchiolitis epidemics onset is in October and November and conclusion varies along time years in hospitalised infants. The most incidence months are January and February with no cases in August and September. It not seems that a late season is followed by an early season in a 2 -year pattern as is described but they alternate between greater numbers of patients with other patients less.

Abstract PO-0184 Table 1 Incidence of bronchiolitis in each epidemic

\begin{tabular}{llllllllllll}
\hline & OCT & NOV & DEC & JAN & FEB & MAR & APR & MAY & JUN & JUL & \\
\hline $2008-09$ & & 2 & 33 & 28 & 6 & & & & & & 69 \\
$2009-10$ & & & 7 & 41 & 48 & 15 & & & & 1 & 112 \\
$2010-11$ & 1 & 1 & 14 & 36 & 35 & 5 & 2 & 1 & & 95 \\
$2011-12$ & 2 & 3 & 31 & 55 & 12 & 5 & 4 & & 1 & 113 \\
& 3 & 6 & 85 & 160 & 101 & 25 & 6 & 1 & 1 & 1 & 389 \\
\hline
\end{tabular}

\section{P0-0185 ACUTE RESPIRATORY TRACT INFECTIONS (ARTI) IN HOSPITALISED CHILDREN: VIRUSES, INTERFERON-ALPHA AND GAMMA, S-IGA LEVELS}

'A Goryaynova, 'I Zakharova, 'L Torshkoeva, 'H Kurbanova, ${ }^{2} \mathrm{~N}$ Koroid, ${ }^{1} \mathrm{I}$ Lagadze. ${ }^{1}$ Pediatrics, Russian Medical Academy of Postgraduate Education, Moscow, Russia; ${ }^{2}$ Pediatrics, Tushino Children's Hospital, Moscow, Russia

10.1136/archdischild-2014-307384.846

Background Detection of respiratory viruses, IFN-alpha, gamma, s-IgA levels in the first 3 years old hospitalised children within 3 days of symptom onset.

Methods The human viruses were detected in nasal wash specimens obtained from 86 children by polymerase chain reaction (PCR), IFN-alpha, gamma, s-IgA plasma and nasal wash specimens levels, IFN-alpha, gamma induced production in vitro were investigated. The IFN-alpha production was induced by
Newcastle disease virus, IFN-gamma production was induced by phytohemagglutinin.

Results Influenza virus B was detected in 35\% investigations, A (H1N1) - 23\%, A(H3N2) - 4\%, Parainfluenza virus (PIV) - 5\%, rhinoviruses (HRV) - 4\%, metapneumovirus (HMPV) - 6\%, bocavirus (HBoV) - 5\%, Adenovirus (AdV) and Respiratory syncytial virus (RSV) - 9\%. Seasonal features were found: A(H1N1) dominated in March (66.7\%), B - in June (77,8\%), PIV was recorded in March only. HRV and RSV were noted as the longest circulation (from January to May), AdV, HBoV, HMPV were detected from March to June. The s-IgA decrease less than $1.5 \mathrm{mg} / \mathrm{ml}$ was found in $75.8 \%$ children in nasal wash specimens. Serum IFN-alpha, gamma were decreasing below a sensitivity threshold (less $2 \mathrm{pg} / \mathrm{ml}$ ) in $67.7 \%$ and $69.4 \%$, especially in influenza children and in ARTI complications. The nasal wash specimens INF-alpha, gamma levels less than $2 \mathrm{pg} / \mathrm{ml}$ were found in $38.7 \%$ and $48.4 \%$. The IFN-alpha, gamma induced levels in vitro were lowered in $22.6 \%$ and $40.3 \%$.

Conclusions Our data demonstrate the IFN-alpha, gamma deficit in children with ARTI. It contributes IFN-alpha, gamma replaceable therapy in infants especially. This is very necessary the ARTI seasonal prevention.

\section{PO-0186 INFLUENCE OF IL-10 GENE POLYMORPHISMS ON IMMUNE RESPONSES AFTER ACELLULAR PERTUSSIS BOOSTER VACCINATION IN ADOLESCENTS}

${ }^{1} \mathrm{~K}$ Gröndahl-Yli-Hannuksela, ${ }^{1} \mathrm{~L}$ Mannermaa, ${ }^{2} \mathrm{~J}$ Mertsola, ${ }^{1} \mathrm{Q}$ He. ${ }^{1}$ Department of Infectious Disease Surveillance and Control, National Institute for Health and Welfare, Turku, Finland; '² Department of Pediatrics, Turku University Hospital, Turku, Finland

\subsection{6/archdischild-2014-307384.847}

Background and aim Despite the mass vaccinations, pertussis has recently caused large epidemics in many industrialised countries. Vaccine-induced immune responses may be impaired due to polymorphisms in genes encoding regulatory cytokines such as IL-10. The aim of this study was to evaluate the role of IL-10 promoter polymorphisms on vaccine responses after acellular pertussis booster vaccinations.

Methods Seventy-five adolescents received diphtheria-tetanusacellular pertussis (dTap) vaccination in 1997. They were followed at 3, 5 and 10 years after. At year 10, a second booster was administrated. Antibodies $(\mathrm{n}=52)$ and cell mediated immunity $(\mathrm{CMI})(\mathrm{n}=38)$ against pertussis antigens were measured. IL-10 polymorphisms (rs1800890 and rs1800896) were detected using Sequenom iPlex Gold system.

Results After the second booster, rs1800896 was found to affect the geometric mean value (GMV) of proliferation (counts per minute, cpm) and stimulation index (SI) against pertussis antigens; pertussis toxin (PT), filamentous hemagglutinin (FHA), pertactin (PRN) (Table).

Another SNP (rs1800890) affected the CMI against PT and FHA after the booster vaccination. Also, the IgG antibody

\begin{tabular}{|c|c|c|c|c|c|c|}
\hline & \multicolumn{3}{|c|}{ Proliferation/cpm } & \multicolumn{3}{|l|}{ SI } \\
\hline & PT & FHA & PRN & PT & FHA & PRN \\
\hline$T / T(n=10)$ & 721,2 & 3651,6 & 1185,5 & 10,3 & 52 & 16,6 \\
\hline$T / C(n=21)$ & 3208,9 & 4902,4 & 1907,8 & 63,7 & 97,3 & 37,8 \\
\hline$C / C(n=4)$ & 112,8 & 665,4 & 196,7 & 1,8 & 10,7 & 3,2 \\
\hline$p$-value & 0,008 & 0,021 & 0,075 & 0,001 & 0,037 & 0,031 \\
\hline
\end{tabular}

